\title{
A REPRESENTATION FOR A CLASS OF LATTICE ORDERED GROUPS
}

\author{
F. D. PEDERSEN( ${ }^{(1)}$
}

In a lattice ordered group ( $l$-group), the set of regular subgroups forms a root system in the complete lattice of all convex $l$-subgroups. Conrad, Harvey, and Holland [4] have shown that an abelian $l$-group can be represented as an $l$-group of real-valued functions on any plenary subset of its root system. This paper is concerned with further investigation of the root system leading to a representation for a class of $l$-groups based on the decomposition of plenary subsets into connected parts (Definition 1). To accomplish this, the concept of $\Gamma$-indecomposable $l$-groups (Definition 8) is introduced. The major theorem (Theorem 15) then presents necessary and sufficient conditions that an l-group be representable as a full subdirect sum of a cardinal sum of $\Gamma$-indecomposable $l$-groups of the first kind.

1. Here we present some of the basic notation, definitions, and theorems relative to the study of $l$-groups. The uninitiated reader might also want to refer to either [1] or [5] whereas a person knowledgeable in this field might prefer to skip this section.

(i) The positive elements of an $l$-group $G$ are denoted by $G^{+}$. From [5, p. 70], it can be deduced that for $g, h \in G^{+}$, there exist $\bar{g}$ and $\bar{h}$ with $\bar{g} \wedge \bar{h}=0$ such that $g=g \wedge h+\bar{g}$ and $h=g \wedge h+\bar{h}$.

(ii) $C(A)$ denotes the convex $l$-subgroup generated by a nonvoid subset $A \subseteq G$. For convenience, $C(\{g\})=C(g)$.

(iii) $\Gamma(G)$ denotes the lattice of all convex $l$-subgroups of $G$. By [3, Introduction], $\Gamma(G)$ forms a complete distributive sublattice of the lattice of all subgroups of $G$. Normal convex $l$-subgroups are called l-ideals. A regular subgroup is an element of $\Gamma(G)$ which is maximal with respect to not containing some $0 \neq g \in G$. For each nonzero $g \in G$, the completeness of $\Gamma(G)$ assures the existence of at least one regular subgroup maximal without containing $g$ [3, Proposition 3.3]. Similarly, if $x \notin K \in \Gamma(G)$, then there exists $H \in \Gamma(G)$ which is maximal without containing $x$ and such that $K \subseteq H$. Thus, as noted in [3], the regular subgroups of $G$ generate the lattice $\Gamma(G)$.

(iv) Given $M \in \Gamma(G), M \neq G, M$ is a prime subgroup if $M$ satisfies any one of the following equivalent conditions [3, Theorem 3.2]:

(a) If $A, B \in \Gamma(G)$ such that $A \cap B \subseteq M$, then $A \subseteq M$ or $B \subseteq M$.

(b) If $a, b \in G^{+}$and $a, b \notin M$, then $a \wedge b \notin M$.

Received by the editors September 14, 1967 and, in revised form, May 28, 1968.

$\left.{ }^{(}\right)$This work is in part a portion of the author's doctoral dissertation written under the direction of Professor A. H. Clifford, Tulane University [6]. 
(c) $M$ is an intersection of a chain of regular subgroups.

(d) The convex $l$-subgroups of $G$ which contain $M$ form a chain.

Also, if $M$ is normal, then each of the above is equivalent to

(e) $G / M$ is totally ordered.

(v) $\Gamma_{0}(G)$ denotes the set of all prime subgroups of $G . \Gamma_{1}(G)$ denotes the set of all regular subgroups of $G$. It follows from property (iv-c) that $\Gamma_{1}(G) \subseteq \Gamma_{0}(G)$.

(vi) A root system is a partially ordered set $S$ with the property that for any $s^{\prime} \in S$, the set of all $s \in S$ such that $s^{\prime} \leqq s$ is a totally ordered subset. $\Gamma_{0}(G)$ and $\Gamma_{1}(G)$ are root systems by property (iv-d).

(vii) For a prime subgroup $M$, it follows from property (iv-b) that if $0 \leqq$ $a \wedge b \in M$, then $a \in M$ or $b \in M$. Thus, as an immediate extension of [4, Lemma 4.5] to the nonabelian case, we have

Lemma 0 . If $0<x$ and $0 \leqq y$ are disjoint elements of $G$ (i.e., $x \wedge y=0$ ) and if $C \in \Gamma_{1}(G)$ is maximal without containing $x$, then $y \in C$ and no $H \in \Gamma_{1}(G)$ maximal without $y$ is contained in $C$.

(viii) A subset $\Delta \subseteq L_{1}(G)$ is plenary if $\Delta$ is an upper class (a lattice dual ideal) in $\Gamma_{1}(G)$ such that $\bigcap\{H: H \in \Delta\}=\{0\}$.

(ix) For $H \subseteq G, H \neq \varnothing$, the polar of $H, H^{*}$, is the set of all $x \in G$ such that $|x| \wedge|y|$ $=0$ for all $y \in H$. $H^{*}$ is a convex $l$-subgroup [5] or [7].

(x) $G$ is a lexicographic extension of $H \in \Gamma(G)$ if

(a) $H$ is normal in $G$;

(b) $x \in G^{+}-H$ implies $y<x$ for all $y \in H^{+}$; and

(c) $G / H$ is totally ordered.

$K \in \Gamma(G)$ is bounded if there exists $x \in G^{+}$such that $y<x$ for all $y \in K^{+}$. If $K \in \Gamma(G)$ is a lexicographic extension of a proper $l$-ideal of itself, and if $K$ is unbounded in $G$, then $G=K \oplus K^{*}[2]$.

(xi) A value of $0 \neq g \in G$ is a regular subgroup which is maximal without containing $g$. $\Gamma g$ denotes the set of all values for $g \in G$. If $0 \neq g \in G$ has only one value, then we call both $g$ and its value special. If $g$ is special with $G_{\alpha}$ as its value, then there exists $N \in \Gamma(G)$ with $C(g)$ a lexicographic extension of $N$ such that $G_{\alpha}=C(g)^{*} \oplus N$. For each $G_{\alpha} \in \Gamma_{1}(G)$, the notation $G^{\alpha}$ refers to $\bigcap\left\{H \in \Gamma(G) ; G_{\alpha}\right.$ is proper in $\left.H\right\}$. Thus, $G^{\alpha}$ is the unique smallest element of $\Gamma(G)$ which properly contains $G_{\alpha}$ [3, Theorem 3.1]. If $g$ is special with $G_{\alpha}$ as its value, then $G^{\alpha}=C(g)^{*} \oplus C(g)$ [3]. $G_{\alpha} \in \Gamma_{1}(G)$ is called essential if there exists $0 \neq g \in G$ such that all values of $g$ are contained in $G_{\alpha}$.

(xii) Using the terminology of (xi), an equivalent definition for a plenary subset $\Delta \subseteq \Gamma_{1}(G)$ is

(a) If $G_{\beta} \in \Delta$ and $G_{\beta} \subseteq G_{\alpha}$, then $G_{\alpha} \in \Delta$; and

(b) Each $0 \neq g \in G$ has a value in $\Delta$ [4].

(xiii) $\Pi\left\{H_{i}: i \in I\right\}$ denotes the large cardinal sum of the l-groups, $H_{i}$, with $I$ as an arbitrary index set. $\Sigma\left\{H_{i}: i \in I\right\}$ denotes the small cardinal sum. Order is 
defined by $0 \leqq x$ if and only if $0 \leqq x_{i}$ for all $i \in I$. A full subdirect sum is a subdirect sum which contains the small cardinal sum. For each $i \in I, \bar{H}_{i}$ denotes the $l$-isomorphic copy of $H_{i}$ in a full subdirect sum of the $H_{2}$ [7]. $G$ is said to be cardinally indecomposable if $G$ can not be represented as a cardinal sum of two nonzero convex $l$-subgroups of $G$.

2. Definition 1. $G_{\alpha}, G_{\beta} \in \Gamma_{1}(G)$ are connected $\left(G_{\alpha} \sim G_{\beta}\right)$ if and only if there exists $G_{\delta} \in \Gamma_{1}(G)$ such that $G_{\alpha}, G_{\beta} \subseteq G_{\delta}$.

PROPOSITION 2. $\sim$ is an equiralence relation on $\Gamma_{1}(G)$.

Proof. For transitivity, let $G_{\alpha} \sim G_{\beta}, G_{\beta} \sim G_{\gamma}$. By definition there exists $G_{o}$ containing $G_{\alpha}, G_{\beta}$ and $G_{\varepsilon}$ containing $G_{\beta}, G_{\gamma}$. Since $\Gamma_{1}(G)$ is a root system, $G_{\delta}$ and $G_{\varepsilon}$ are comparable. Thus, $\max \left\{G_{\delta}, G_{\varepsilon}\right\}$ contains $G_{\alpha}, G_{\gamma}$.

Definition 3. A connected part of $\Gamma_{1}(G)$ is an equivalence class of $\Gamma_{1}(G)$ under $\sim$ and will be denoted by $\Gamma_{i}$.

Note that if we restrict $\sim$ to a plenary subset $\Delta \subseteq \Gamma_{1}(G)$, then the resulting classes will be of the form $\Delta_{1}=\Gamma_{i} \cap \Delta$ for all $i$ such that $L_{i} \cap \Delta \neq \delta$. It should also be noted that the $\Delta_{i}$-classes are maximal directed subsets.

Definition 4. (a) $\Delta$, a plenary subset, is said to be connected if there is only one equivalence class. (In other words, a plenary subset $\Delta$ is connected if and only if $\Delta$ is directed.)

(b) $G$ is $\Gamma$-indecomposable if there exists a plenary subset of $\Gamma_{1}(G)$ which is connected.

(c) An element $D$ in $\Delta_{i}$ is dominating with respect to $\Delta_{i}$ if for each $G_{\alpha}$ in $\Delta_{i}$ either $D \subseteq G_{\alpha}$ or $G_{\alpha} \subseteq D$.

(d) $G$ is $\Gamma$-indecomposable of the first kind if there exists a connected plenary subset of $\Gamma_{1}(G)$ which contains a dominating element.

(e) $G$ is $\Gamma$-indecomposable of the second kind if $G$ is $\Gamma$-indecomposable but there does not exist a connected plenary subset of $\Gamma_{1}(G)$ with a dominating element.

THEOREM 5. If $G$ is $\Gamma$-indecomposable, then $G$ is cardinally indecomposable.

Proof. Assume $G=A \oplus B$. Since any $G_{\alpha}$ which is regular is also prime, and since $A \cap B=\{0\} \subseteq G_{\alpha}$, it follows that $G_{\alpha}$ contains either $A$ or $B$ but not both. We need only consider positive elements of $G$; and any $g \in G^{+}$can be expressed uniquely as $x+y$ where $0 \leqq x \in A, 0 \leqq y \in B$. Since any $x \in A^{+}$is disjoint from any $y \in B^{+}$, Lemma 0 quoted in $\$ 1$ may be applied; and, thus, the values for $x \in A$ are noncomparable to the values for $y \in B$. Thus, any plenary subset $\Delta$ can be written as the union of two disjoint sets, $\Delta_{1}$ and $\Delta_{2}$, such that $\Delta_{1}$ contains values of elements in $A$ and $\Delta_{2}$ contains values of elements in $B$. But, since $\Delta$ is assumed to be connected, $\Delta$ is contained in one of $\Delta_{1}$ or $\Delta_{2}$. Thus, either the elements of $A$ or the elements of $B$ have no values in $\Delta$, which implies that either $A$ or $B$ is zero since $\Delta$ is plenary.

Definition 6. $0<g \in G$ is a weak unit if $0<h \wedge g$ for all $h \in G^{+}$.

A nonunit is an element $0<x$ for which there exists $0<y$ such that $x \wedge y=0$. 
THEOREM 7. If $G$ is an l-group, then the following are equivalent:

(a) $G$ is $\Gamma$-indecomposable of the first kind;

(b) $G$ contains a weak unit which is special;

(c) $\Gamma_{1}(G)$ contains a dominating element;

(d) $G$ is a lexicographic extension of a proper l-ideal.

Proof. (a) $\rightarrow$ (b). Let $\Delta$ be a connected plenary subset of $\Gamma_{1}(G)$ with dominating element, $G_{\alpha}$. Since the values of $0<x \in G^{\alpha}-G_{\alpha}$ with respect to $\Delta$ form a trivially ordered subset of $\Delta, x$ has only $G_{\alpha}$ as value in $\Delta$; and, thus, $x$ is special [4, Theorem 3.7]. Assume $y \wedge x=0$. It follows from the lemma quoted in $\S 1$ that $y$ has no value comparable to $G_{\alpha}$. Since $G_{\alpha}$ is dominating, $y$ has no value in $\Delta$; and, thus, $y=0$. Therefore, $x$ is a weak unit by definition.

(b) $\rightarrow$ (c). Let $x$ be a weak unit which is also special with $G_{\alpha}$ as its only value. Then, $G_{\alpha}=C(x)^{*} \oplus N$ where $N$ is the maximal convex $l$-subgroup of $C(x)$. Since $x$ is a weak unit, $C(x)^{*}=\{0\}$; and, thus, $G_{c}=N \subset C(x)$. Let $G_{\beta} \in \Gamma_{1}(G)$. If $x \notin G_{\beta}$, then $x$ has a value containing $G_{\beta}$. Since $G_{c}$ is the only value for $x, G_{\beta} \subseteq G_{\text {cis }}$. If $x \in G_{\beta}$, then $G_{u} \subseteq C(x) \subseteq G_{\beta}$. Hence $G_{\alpha}$ is dominating in $\Gamma_{1}(G)$.

(c) $\rightarrow(\mathrm{d})$. Let $M_{0}$ be the convex $l$-subgroup generated by all nonunits. $M_{0}$ has the following properties [3, p. 111]:

(1) $G$ is a lexicographic extension of $M_{0}$;

(2) $M_{0}$ is a prime $l$-ideal or $M_{0}=G$;

(3) $M_{0}$ is the smallest convex $l$-subgroup of $G$ that is comparable to all convex l-subgroups of $G$.

Let $G_{\alpha}$ be a dominating element of $\Gamma_{1}(G)$. Using the properties of $M_{0}$, it suffices to show that $G_{\alpha}$ is comparable to all convex l-subgroups of $G$. Let $K \in \Gamma(G)$; and assume $K \notin G_{\alpha}$. For $x \in G_{\alpha}^{+}-K, x \notin K$ implies there exists a value for $x$, say $G_{\beta}$, such that $G_{\beta} \supseteq K$. Since $G_{\alpha}$ is dominating in $\Gamma_{1}(G), G_{\alpha}$ is comparable to $G_{\beta}$. Thus, $x \in G_{\alpha}$ and $x \notin G_{\beta}$ imply that $G_{\beta} \subset G_{\alpha}$. Thus, $K \subseteq G_{\beta} \subset G_{\alpha}$ or $K \subset G_{\alpha}$.

(d) $\rightarrow$ (a). If $G$ is a lexicographic extension of a proper $l$-ideal $M$, then $M \supseteq M_{0}$ (notation as in preceding proof); and moreover, there exists $G_{\alpha} \in \Gamma_{1}(G)$ with $G_{\alpha} \supseteq M_{0}$. Therefore, $G_{\alpha}$ is comparable to all convex $l$-subgroups of $G$. Thus $G_{\alpha}$ is dominating in $\Gamma_{1}(G)$; and $\Delta=\Gamma_{1}(G)$ satisfies condition (a).

In general, a maximal chain in a root system is not cofinal; but a maximal chain in a connected part, $\Delta_{i}$, will be cofinal in $\Delta_{i}$.

THEOREM 8. $\Gamma_{1}(G)$ is connected and its special elements form a cofinal subset if and only if $G=\bigcup\left\{G_{i}: i \in \Lambda\right\}$ where $\left\{G_{1}: i \in \Lambda\right\}$ is a chain of convex l-subgroups each of which is $\Gamma$-indecomposable of the first kind.

Proof. Assume that $\Gamma_{1}(G)$ is connected and that its special elements are cofinal in $\Gamma_{1}(G)$. For each special $G_{i}$, choose $0<g_{i}$, special, and with $G_{i} \in \Gamma g_{i}$. Note that $G_{i} \cap C\left(g_{i}\right)=M_{i}$ is the maximal convex $l$-subgroup of $C\left(g_{i}\right)$; and, moreover, $C\left(g_{i}\right)$ is a lexicographic extension of $M_{i}$. Thus, Theorem 6 implies that each $C\left(g_{i}\right)$ is $\Gamma$-indecomposable of the first kind. It will now be shown that $G$ is the union of any 
$\left\{C\left(g_{i}\right): i \in I\right\}$ where the corresponding $\left\{G_{i}: i \in I\right\}$ is a chain of special $G_{i}$ 's which is cofinal in $\Gamma_{1}(G)$. Suppose $G_{i}$ is proper in $G_{j}$ and $g_{i} \notin C\left(g_{j}\right)$; then, since $G_{i}$ is the only value for $g_{i}, C\left(g_{j}\right) \subseteq G_{i} \subset G_{j}$-a contradiction. Therefore, $g_{i} \in C\left(g_{j}\right)$, or $C\left(g_{i}\right) \subset C\left(g_{j}\right)$. Thus, $\left\{C\left(g_{i}\right): i \in I\right\}$ is chain order isomorphic to $\left\{G_{i}: i \in I\right\}$. Let

$$
K=\bigcup\left\{C\left(g_{i}\right): i \in I\right\} .
$$

Then $K$ is a convex l-subgroup of $G$. If $K \neq G$, there exists $G_{\alpha} \in \Gamma_{1}(G)$ such that $G_{\alpha} \supseteq K$. Since $\left\{G_{i}: i \in I\right\}$ is cofinal in $\Gamma_{1}(G), G_{\alpha} \subseteq G_{i}$ for some $i \in I$. This implies that $g_{i} \notin K$-contrary to the definition of $K$.

Conversely, assume that $G=\bigcup\left\{G_{i}: i \in \Lambda\right\}$ where each $G_{i}$ is a convex $l$-subgroup of $G$ which is $\Gamma$-indecomposable of the first kind and $\left\{G_{i}: i \in \Lambda\right\}$ is a chain. For $0<x \in G, x \in G_{i}$ for some $G_{i}$; and there exists a $y$ such that $x \leqq y$ and $y$ is a special element which has a special value $G_{y}^{\prime}$ which is dominating in $\Gamma_{1}\left(G_{i}\right)$. Since $y$ is special, the convex $l$-subgroup generated by $y$ in $G_{i}$ is a lexicographic extension of a proper $l$-ideal. Since $G_{i}$ is convex in $G$, the convex $l$-subgroup generated by $y$ is the same for $G$ as it is for $G_{i}$. Thus, $C(v)$ is a lexicographic extension of a proper $l$-ideal. Therefore, $y$ is special with value $G_{y} \in \Gamma_{1}(G)$. This implies that all values of $x$ in $\Gamma_{1}(G)$ are contained in some special $G_{y}$-which, in turn, implies that the special elements are cofinal. To show $\Gamma_{1}(G)$ is connected it suffices to show two special elements $G_{\alpha}, G_{\delta}$ are connected since they are cofinal in $\Gamma_{1}(G)$. Choose $z_{1}, z_{2}>0$, special, such that $G_{\alpha} \in \Gamma z_{1}$ and $G_{\delta} \in \Gamma z_{2}$. Since $G$ is the union of the $G_{i}$, some $G_{j}$ contains both $z_{1}$ and $z_{2}$. $G_{j}$ is assumed to be $\Gamma$-indecomposable; thus, there exists $z_{3}>0$ in $G_{j}$ such that $z_{3}$ is special and $z_{3} \geqq z_{1}, z_{2}$. But, the condition that $z_{3} \geqq z_{1}, z_{2}$ immediately implies that the values for $z_{1}$ and the values for $z_{2}$ are contained in the value for $z_{3}$. Thus, if $G_{\beta}$ is the value for $z_{3}$ in $\Gamma_{1}(G)$, then $G_{\beta^{\prime}} \supseteq G_{\alpha}, G_{\delta}$-or any two special elements of $\Gamma_{1}(G)$ are connected.

3. Definition 9. Let $\Delta$ be a plenary subset of $\Gamma_{1}(G)$ and $\Delta_{i}$ a connected part of $\Delta$. Then the union of the set of all $x$ having all values with respect to $\Delta$ in $\Delta_{i}$, together with zero, is denoted by $H_{i}$; that is

$$
H_{i}=\left\{x \in G: G_{\alpha} \in \Gamma x \cap \Delta \rightarrow G_{\alpha} \in \Delta_{i}\right\} \cup\{0\} .
$$

Proposition 10. $H_{i}$ is a convex l-subgroup of $G$; and, moreover,

$$
H_{\imath}=\bigcap\left\{G_{\delta}: G_{\delta} \in \Delta-\Delta_{i}\right\} .
$$

Proof. Since the set of all convex $l$-subgroups forms a complete lattice, it need only be shown that $H_{i}=\bigcap\left\{G_{\delta}: G_{\delta} \in \Delta-\Delta_{i}\right\}$. Let $x \in H_{i}$ and $G_{\delta} \in \Delta-\Delta_{i}$. If $x \notin G_{\delta}$, then $\Delta$ plenary implies that there exists a value $G_{\alpha}$ of $x$ in $\Delta$ such that $G_{\alpha} \supseteq G_{\delta}$. But $\Delta-\Delta_{i}$ being an upper class implies $G_{\alpha} \notin \Delta_{i}$, or $x \notin H_{i}$. Hence $x \in G_{\delta}$. Conversely, if $x \notin H_{i}$, then $x$ has a value $G_{\delta} \in \Delta-\Delta_{i}$, and $x \notin G_{\delta}$.

We shall use the following properties from the notes of P. F. Conrad (proofs may be found in [6, Appendix]); 
(1) If $G_{H_{i}}=\left\{M: M \in \Gamma_{0}(G)\right.$ and $\left.M \notin H_{i}\right\}$, then $\sigma: G_{H_{i}} \rightarrow \Gamma_{0}\left(H_{i}\right)$, defined by $\sigma(M)=M \cap H_{i}$, is a one to one and onto mapping.

(2) $G_{\alpha} \in G_{H_{i}} \cap \Gamma_{1}(G)$ if and only if $\sigma\left(G_{\alpha}\right) \in \Gamma_{1}\left(H_{i}\right)$. Furthermore, $G_{\alpha} \in \Gamma x \cap G_{H_{i}}$, $x \in H_{i}$, if and only if $\sigma\left(G_{\alpha}\right)$ is a value for $x$ in $\Gamma_{1}\left(H_{i}\right)$.

Proposition 11. $\sigma$ is one to one from $\Delta_{i}$ to $\Gamma_{1}\left(H_{i}\right)$ if and only if $G_{\alpha} \in \Delta_{i}$ implies $G_{\alpha} \neq H_{i}$. If this is the case, $\sigma\left(\Delta_{i}\right)$ is a plenary subset of $\Gamma_{1}\left(H_{i}\right)$.

Proof. We need only show that under these conditions the image of $\Delta_{i}$ is plenary. Let $0<x \in H_{i}$; then all the values of $x$ in $\Delta$ are in $\Delta_{i}$. Thus, there exists $G_{\alpha} \in \Gamma x \cap \Delta_{i}$ with $\sigma\left(G_{\alpha}\right)$ as a value for $x$ in $\Gamma_{1}\left(H_{i}\right)$-property 2, above. Suppose $\sigma\left(G_{\alpha}\right) \in \Gamma_{1}\left(H_{i}\right)$ and $0<x \notin \sigma\left(G_{\alpha}\right)$ for $x \in H_{i}$. Then $x \notin G_{\alpha}$, and there exists $G_{\beta} \in \Delta_{i}$ such that $G_{\beta} \in \Gamma x$, $G_{\alpha} \subseteq G_{\beta}$. By property 2 above, $\sigma\left(G_{\beta}\right)$ is a value for $x$ in $\Gamma_{1}\left(H_{i}\right)$. Since $G_{\alpha} \subseteq G_{\beta}$, $\sigma\left(G_{\alpha}\right) \subseteq \sigma\left(G_{\beta}\right)$. Therefore $\sigma\left(\Delta_{i}\right)$ is plenary in $\Gamma_{1}\left(H_{i}\right)$.

Corollary 12. If no $G_{\alpha}$ in $\Delta_{i}$ contains $H_{i}$, then $H_{i}$ is $\Gamma$-indecomposable.

Proposition 13. If no $G_{\alpha}$ in $\Delta_{i}$ contains $H_{i}$, then $H_{i}$ is unbounded in $G$.

Proof. Assume $H_{i}$ is bounded. Thus, there exists $x$ in $G^{+}$such that $y<x$ for all $y \in H_{i}^{+}$. Since $\Delta$ is plenary, $\Delta_{i}$ contains a value $G_{\alpha}$ for $x$. By hypothesis, $G_{\alpha}$ does not contain $H_{i}$; and hence, there exists $y \in H_{i}^{+}-G_{\alpha}$. Consider first the case where $G_{\alpha}$ is maximal in $\Delta_{i}$. Then $y$ is special; and $G_{\alpha}=C(y)^{*} \oplus N$ where $N$ is the maximal $l$-ideal of $C(y)$. Moreover, $G^{\alpha}=C(y)^{*} \oplus C(y)$. Then, $0<x \in G^{\alpha}$ implies the existence of $0 \leqq x_{1} \in C(y)^{*}, 0 \leqq x_{2} \in C(y)$, such that $x_{1}+x_{2}=x$; and $x \notin C(y)^{*}$ implies that $0<x_{2}$. Moreover, since $x_{2} \in C(y)$, there exists $n>0$ such that $0<x_{2}<n y$. Thus, using the fact that $x_{1} \wedge n y=0, n y=x \wedge n y=\left(x_{1}+x_{2}\right) \wedge n y=\left(x_{1} \wedge n y\right)+\left(x_{2} \wedge n y\right)=x_{2}$, which is a contradiction. Assume, on the other hand, that $G_{\alpha}$ is not maximal in $\Delta_{i}$. Then, there exists $G_{\beta} \in \Delta_{i}$ with $G^{\alpha} \subseteq G_{\beta}$. By hypothesis, there exists $z \in H_{i}^{+}-G_{\beta}$. Since $z<x$ implies the contradiction $z \in G^{\alpha} \subseteq G_{\beta}, H_{i}$ must be unbounded.

Proposition 14. If $H_{i}$ is unbounded and $\Delta_{i}$ contains a dominating element, then $G=H_{i} \oplus H_{i}^{*}$. Moreover, $H_{i}$ is a proper lexicographic extension of an l-ideal; and the mapping $\sigma$ from $\Delta_{i}$ to $\Gamma_{1}\left(H_{i}\right)$ is one to one.

Proof. Let $G_{\alpha}$ be a dominating element of $\Delta_{i}$. It will be shown that $H_{i}$ is not contained in $G_{\alpha}$. This fact immediately implies that no element of $\Delta_{i}$ contains $H_{i}$-or that the mapping from $\Delta_{i}$ to $\Gamma_{1}\left(H_{i}\right)$ is one to one. Thus, the image of $\Delta_{i}$ will provide $H_{i}$ with a connected plenary subset with dominating element; and $H_{i}$ is, therefore, a proper lexicographic extension of an $l$-ideal. Then, by applying the theorem mentioned in $\S 1$, part (x), $G=H_{i} \oplus H_{i}^{*}$ can be concluded.

Assume that $H_{i} \subseteq G_{\alpha}$. Let $0<g \in G^{\alpha}-G_{\alpha}$. Since $H_{i}$ was assumed unbounded, there exists $0<h \in H_{i}$ such that $h \$ g$. Also $h \ngtr g$ since this would imply that $g \in H_{i} \subseteq G_{\alpha}$, contradicting the choice of $g$. Thus, $g$ and $h$ are not comparable. Let $g=g \wedge h+\bar{g}, h=g \wedge h+\bar{h}$ with $\bar{h} \wedge \bar{g}=0$. The incomparability of $g$ and $h$ implies, moreover, that $\bar{g}$ and $\bar{h}$ are greater than 0 . Since $g \wedge h \in H_{i} \subseteq G_{\alpha}, \bar{g} \in G^{\alpha}-G_{\alpha}$; and, 
thus, $\bar{h} \wedge \bar{g}=0$ implies that $\bar{h}$ has no value comparable to $G_{\alpha}$. But, $\bar{h} \in H_{i}$ implies that all values of $\bar{h}$ in $\Delta$ are comparable to the dominating element $G_{\alpha}$ of $\Delta_{i}$; a contradiction.

THEOREM 15. If $G$ is an l-group, then the following are equivalent:

(a) there exists $\Delta$ plenary such that each $\Delta_{i}$ contains a cofinal chain of dominating special elements;

(b) there exists $\Delta$ plenary such that each $\Delta_{i}$ contains a dominating element and each $H_{i}$ is unbounded;

(c) there exists $\Delta$ plenary such that for each $i, G=H_{i}^{*} \oplus H_{i}$ and $H_{i}$ is $\Gamma$-indecomposable of the first kind;

(d) $G$ is l-isomorphic to a full subdirect sum of $\Gamma$-indecomposable l-groups of the first kind.

It is to be noted that the $\Gamma$-indecomposable $l$-groups mentioned in (d) are the $H_{i}$ obtained from the choice of $\Delta$ as in (a) or (b). The proof of Theorem 15 depends upon the following lemma-the proof of which will appear later in this section.

LeMmA 16. If $\Pi=\Pi\left\{G_{i}: i \in I\right\}$, where each $G_{i}$ is an l-group, and if $G$ is a full subdirect sum of $\Pi$, then each $G_{\alpha} \in \Gamma_{1}(G)$ is classified by means of the projections $\pi_{i}$ from $G$ onto the $G_{i}$ into one of the following two types:

[first type]: there exists one $G_{j}$ such that $\pi_{i}\left(G_{\alpha}\right)=G_{i}$ for all $i \neq j, \pi_{j}\left(G_{\alpha}\right)$ is regular in $G_{j}$, and $G_{\alpha}=\left(\bar{G}_{j} \cap G_{\alpha}\right) \oplus \bar{G}_{j}^{*}$.

[second type]: $\pi_{i}\left(G_{\alpha}\right)=G_{i}$ for all $i$.

Moreover, the set of all $G_{\alpha}$ of the first type is plenary, and no $G_{\beta}$ of the second type is connected to a $G_{\alpha}$ of the first type.

Proof of Theorem 15. (a) $\rightarrow$ (b). It need only be shown that $H_{i}$ is unbounded or, by Proposition 13, that no $G_{\alpha}$ in $\Delta_{i}$ contains $H_{i}$. By hypothesis on $\Delta_{i}$, it suffices to show that no dominating special element $G_{\beta}$ in $\Delta_{i}$ contains $H_{i}$. Let $0<x \in G$ whose only value is $G_{\beta}$, then $x \in H_{i}$. Since $x \notin G_{\beta}$, we can conclude that $G_{\beta} \notin H_{i}$.

(b) $\rightarrow$ (c). This follows from Proposition 14 .

(c) $\rightarrow$ (d). Let $\Delta$ be chosen as in (c). Then each $g \in G$ has a unique expression as $g^{i}+g_{i}, g^{i} \in H_{i}^{*}, g_{i} \in H_{i}$. Define $\phi_{i}$ by $\phi_{i}(g)=g_{i}$. Then $\phi_{i}$ is an $l$-homomorphism for each $i$. Let $\phi$ denote the induced $l$-homomorphism from $G$ to $\Pi\left\{H_{i}: i \in I(\Delta)\right\}$. We need only show that $\phi$ is one to one. If $\phi(g)=0, g \geqq 0$, then $g_{i}=0$ for all $i$. Let us now assume that the values for $x \in H_{i}, i$ in the index set of $\lrcorner$, are cofinal in $\Delta_{i}$ for each $i$. The assumption will be proved in the following paragraph. Suppose $g \neq 0$, then $g$ has a value $G_{\beta} \in \Delta_{i}$ for some $i$. From the foregoing assumption, $G_{\beta} \subseteq G_{\alpha} \in \Gamma x$, $0<x \in H_{l}$. Thus, $0<x \wedge g=x \wedge\left(g^{i}+g_{i}\right)=\left(x \wedge g^{i}\right)+\left(x \wedge g_{i}\right)$ which implies $g_{i}>0$ since $g^{i} \wedge x=0$ - a contradiction. $g=0$ implies $\phi$ is one to one and an $l$-isomorphism into. Since for each $g_{i} \in H_{i} \subseteq G, \phi\left(g_{i}\right)$ is equal to the element 0 in all except the $i$ th coordinate and is $g_{i}$ in the $i$ th coordinate, $\phi(G)$ contains $\Sigma \oplus H_{i}$. 
Since $H_{i}$ is $\Gamma$-indecomposable of the first kind, by Theorem 7 there exists $G_{\gamma} \in \Gamma_{1}\left(H_{i}\right)$ such that $G_{\gamma}$ is a value for a special element $0<x \in H_{i}$. As in Proposition 11, $\sigma\left(G_{\alpha}\right)=G_{\alpha} \cap H_{i}=G_{\gamma}$ and $G_{\alpha}$ is the only value for $x$ in $G$. Suppose $G_{\beta} \supseteq G_{\alpha}$ and $G_{\beta} \in \Gamma y, y>0$. Let $y=y^{i}+y_{i}, 0 \leqq y^{i} \in H_{i}^{*}$ and $0 \leqq y_{i} \in H_{i}$. Since $y^{i} \wedge x=0, y^{i}$ can have no value comparable to $G_{\alpha}$. Thus, $G_{\beta} \in \Gamma y_{i}, y_{i} \in H_{i}$; or, the values for $x \in H_{i}$ are cofinal in $\Delta_{i}$.

$(d) \rightarrow(a)$. Assume that $G$ is a full subdirect sum of $\Gamma$-indecomposable $l$-groups, $E_{i}, i \in I$, each of which is $\Gamma$-indecomposable of the first kind. By Lemma 16, the regular subgroups of the first type form a plenary subset $\Delta$ of $\Gamma_{1}(G)$. Moreover, $\Delta_{i}=\left\{G_{\alpha}: \pi_{i}\left(G_{\alpha}\right) \in \Gamma_{1}\left(E_{i}\right)\right\}$ is just the set of all $G_{\alpha} \in \Gamma_{1}(G)$ such that $G_{\alpha} \notin \bar{E}_{i}$. Moreover, $\bigcup\left\{\Delta_{i}: i \in I\right\}$ is $\Delta$. Thus the mapping $G_{\alpha} \rightarrow G_{\alpha} \cap \bar{E}_{i}$ is a one to one, onto, order preserving mapping from $\Delta_{i}$ to $\Gamma_{1}\left(\bar{E}_{i}\right)$. Since $E_{i}$ is $l$-isomorphic to $\bar{E}_{i}, \Delta_{i}$ is connected with a cofinal sequence of dominating special elements by Theorem 7 . Since any two elements from different $\Delta_{i}$ will necessarily be not comparable, $\Delta$ satisfies condition (a).

Proof of Lemma 16. Assume $G_{\alpha}$ is a regular subgroup of $G$ which is not of the second type. The negation of the second type implies that there exists $i \in I$ such that $\pi_{i}\left(G_{\alpha}\right) \neq G_{i}$. Moreover, since $G$ contains the small sum it can be extracted from [7] that $G=\bar{G}_{i} \oplus \bar{G}_{i}^{*}$. Since $G_{\alpha}$ is not of the second type $G_{\alpha} \neq \bar{G}_{i}$; and, thus, $\bar{G}_{i}^{*} \subseteq G_{\alpha}$ since $G_{\alpha}$ is prime. Hence, $G_{\alpha}=\left(G_{\alpha} \cap \bar{G}_{i}\right) \oplus \bar{G}_{i}^{*}$. Thus $\pi_{i}\left(G_{\alpha}\right)=G_{\alpha} \cap \bar{G}_{i}=\sigma\left(G_{\alpha}\right)$, in the notation of Proposition 11, and so is regular. The conclusion that no first type element is connected to a second type element follows from the fact that $G=G_{\alpha} \vee G_{\beta}$ where $G_{\alpha}$ is of the first type and $G_{\beta}$ is of the second type.

The following corollary illustrates an alternative way of looking at Theorem 7.2 of [2]. Since a proof of the corresponding theorem is given in [2], the proof will not be given here. Before stating the corollary we need two definitions.

Definition 17. $0<b \in G$ is a basic element if $C(b)$ is totally ordered.

Definition 18. $G$ has a basis if for every $0<x \in G$ there exists a basic element $b$ such that $0<b \leqq x$. The basis group for $G$ is the convex $l$-subgroup generated by the basic elements in $G$.

COROLLARY 19. If $G$ is an l-group, then the following are equivalent:

(a) G has a basis and no maximal convex o-subgroup of the basis group is bounded;

(b) there exists $\Delta$ plenary such that each $\Delta_{i}$ is a chain of special elements of $\Gamma_{1}(G)$;

(c) $G$ is l-isomorphic to a full subdirect sum of a cardinal sum of o-groups.

It is noted that the $o$-groups referred to in (c) are the $H_{i}$ derived from a choice of $\Delta$ as in (b).

4. Definition 20. For a plenary set $\Delta$, the $\Delta_{i}$-subgroup $M_{i}$ is the convex $l$-subgroup formed by intersecting the elements of $\Delta_{i}$; i.e., $M_{i}=\bigcap\left\{G_{\alpha}: G_{\alpha} \in \Delta_{i}\right\}$.

Proposition 21. If $M_{i}$ is a normal $\Delta_{\imath}$-subgroup, then $G / M_{i}$ is $\Gamma$-indecomposable. 
Proof. The natural $l$-homomorphism of $G$ onto $G / M_{i}$ preserves the lattice structure of the set of all $H \in \Gamma(G)$ such that $H \supseteq M_{i}$. Thus, $\Delta_{i}$ is mapped onto a plenary set for $G / M_{i}$ and is connected.

Proposition 22. Eiery maximal element of $\Gamma(G)$ is normal if and only if each connected part of $\Gamma_{1}(G), \Gamma_{i}$, is invariant under inner automorphisms of $G$.

Proof. First assume all maximal elements of $\Gamma(G)$ are normal. Let $G_{\alpha} \in \Gamma_{i}$ and $0<x$ with $x+G_{\alpha}-x \neq G_{\alpha}$. Thus, $x \notin G_{\alpha}$ which implies that there exists $G_{\beta} \in \Gamma_{x}$ such that $G_{\alpha} \subseteq G_{\beta}$. If $G_{\beta}$ is maximal in $\Gamma(G)$, then $x+G_{\beta}-x=G_{\beta} \supseteq G_{\alpha}$ and $x+G_{\alpha}-x$. Thus, $x+G_{\alpha}-x \in \Gamma_{i}$. If $G_{\beta}$ is not maximal in $\Gamma(G)$, then there exists $G_{\gamma} \supset G_{\beta}$ and $x \in G_{\gamma}$. Thus, $x+G_{\gamma}-x=G_{\gamma} \supseteq G_{\alpha}$ and $x+G_{\alpha}-x$ which implies that $x+G_{\alpha}-x \in \Gamma_{i}$. For the converse assume $G_{\alpha}$ is maximal in $\Gamma(G)$. Thus, $G_{\alpha}$ is the maximal element of some $\Gamma_{i}$. Since $\Gamma_{i}$ is invariant, for each $x \in G,-x+G_{\alpha}+x \in \Gamma_{i}$ and, thus, $G_{\alpha}$ $\supseteq-x+G_{\alpha}+x$. Since $x$ was arbitrary $G_{\alpha}$ is normal.

Proposition 23. If the maximal elements of $\Gamma(G)$ are normal, then the $\Gamma_{i}$ subgroups $M_{i}$ are all normal.

Proof. Suppose $y \in M_{i}$ and $x+y-x \notin M_{i}$. Then $x+y-x$ has a value in $\Gamma_{i}$ since $M_{i}=\bigcap\left\{G_{\alpha} \in \Gamma_{i}\right\} . y \in M_{i}$ implies $y$ does not have a value in $\Gamma_{i}$. Let $G_{\alpha} \in \Gamma_{i}$ such that $G_{\alpha} \in \Gamma x+y-x$. Thus, $-x+G_{\alpha}+x$ does not contain $y$ which implies that $-x+G_{\alpha}$ $+x \in \Gamma_{j}$ for $j \neq i$. Therefore $\Gamma_{i}$ is not invariant under inner automorphisms and this contradicts Proposition 22.

THEOREM 24. If $\left\{M_{j}: j \in J\right\}$ is a collection of normal $\Delta_{i}$-subgroups such that $\bigcap\left\{M_{i}: j \in J\right\}=\{0\}$, then $G$ is l-isomorphic to a subdirect sum of $\Gamma$-indecomposable l-groups.

Proof. The natural homomorphism from $G$ to $G / M_{j}$ is an onto $l$-homomorphism, for each $j \in J$. Thus, the induced mapping $x \rightarrow x+M_{j}$ will be an $l$-homomorphism of $G$ onto a subdirect sum of the $G / M_{j}$. Since $\cap\left\{M_{j}: j \in J\right\}=\{0\}$, this mapping will be one to one.

COROllary 25. If $G$ has a collection of normal primes $N_{t}, t \in T$ such that $\bigcap\left\{N_{t}: t \in T\right\}=\{0\}$, then $G$ is l-isomorphic to a subdirect sum of $\Gamma$-indecomposable l-groups.

Proof. Let $\Delta=\left\{G_{\alpha} \in \Gamma_{1}(G): G_{\alpha} \supseteq N_{t}\right.$ for some $\left.t \in T\right\} . \Delta$ is plenary; and any $\Delta_{i}$-subgroup, $M_{i}$, will be an intersection of a subcollection of the $N_{t}$. Thus, the $\Delta_{i}$-subgroups will be normal and $\bigcap\left\{M_{i}: M_{i}\right.$ the $\Delta_{i}$-subgroup $\}=\bigcap\left\{N_{t}: t \in T\right\}$ $=\{0\}$. Now, apply Theorem 24 .

It is relatively easy to exhibit a counterexample to show that the converse of Corollary 25 is not true. Thus, since the hypothesis of the corollary is trivially equivalent to representability as a subdirect sum of $o$-groups it follows that representability as a subdirect sum of $o$-groups implies representability as a subdirect sum of $\Gamma$-indecomposable $l$-groups with the converse false. 
CoRollary 26. If the maximal elements of $\Gamma(G)$ are normal, then $G$ is l-isomorphic to a subdirect sum of $\Gamma$-indecomposable l-groups.

Proof. Apply Propositon 23 and Theorem 24.

\section{BIBLIOGRAPHY}

1. G. Birhoff, Lattice theory, rev. ed., Amer. Math. Soc. Colloq. Publ., Vol. 25, Amer. Math. Soc., Providence, R. I., 1948.

2. P. F. Conrad, Some structure theorems for lattice ordered groups, Trans. Amer. Math. Suc. 99 (1961), 212-240.

3. - The lattice of all convex l-subgroups of a lattice ordered group, Czechoslovak Math. J. 15 (1965), 101-123.

4. P. F. Conrad, J. Harvey and C. Holland, The Hahn embedding theorem for abelian lattice ordered groups, Trans. Amer. Math. Soc. 108 (1963), 143-169.

5. L. Fuchs, Partially ordered algebraic systems, Addison-Wesley, Reading, Mass., 1963.

6. F. Pedersen, Contributions to the theory of regular subgroups and prime subgroups of a lattice ordered group, Tulane Dissertation, New Orleans, La., 1966.

7. F. Sik, Zur Theorie der halbegeordneten Gruppen, Czechoslovak Math. J. 6 (1956), 1-25. (Russian. German summary)

SOUTHERN ILLINOIS UNIVERSITY,

Carbondale, Illinois 